\title{
Using Syndromic Surveillance Data to Describe Chronic High Frequency ED Utilization
}

\author{
Erin E. Austin* \\ Division of Surveillance and Investigation, Virginia Department of Health, Richmond, VA, USA
}

\section{Objective}

Leverage existing syndromic surveillance data to characterize the population of chronic high frequency emergency department (ED) users and to understand the health complaints for which this population utilizes emergent health care services.

\section{Introduction}

Utilization and overcrowding of EDs has been a prominent component of the health care reform debate in the United States for the last several years. In Virginia, the ED utilization rate has increased $27.5 \%$ between 2000 and 2012 from 34.5 visits to 44.0 visits per 100 persons ${ }^{1}$. Individuals with high frequency utilization of EDs account for a disproportionate number of visits, which can place burden on already strained health care resources ${ }^{2}$. This study aims to use existing syndromic surveillance data received electronically by the Virginia Department of Health (VDH) to describe demographic and utilization characteristics among chronic high frequency ED users in order to better understand the health complaints affecting this population.

\section{Methods}

A retrospective study was conducted using syndromic surveillance data received by VDH from 44 acute care hospital EDs between January 1, 2012 and December 31, 2013. A chronic high frequency $\mathrm{ED}$ user was defined as a person with more than $10 \mathrm{ED}$ visits in 2012 and more than 10 ED visits in 2013 at the same acute care hospital based on unique medical record number (MRN). Analyses were conducted at the patient level based on MRN to characterize population demographics and at the visit level based on chief complaint (CC) to characterize the health complaints of chronic high frequency ED users.

\section{Results}

The study identified 1,336 chronic high frequency ED users. Median and mean number of ED visits per user were 33 and 39, respectively (range 22-392), for a total of 52,006 ED visits over the 2 year period. Chronic high frequency users represented $0.1 \%$ of the ED patient population served during the two year study period but $1.4 \%$ of the total 3.7 million ED visits. Nearly two-thirds (64.4\%) of the chronic users were female, and female users were significantly younger than males, with a mean age of 41.4 years compared to 45.8 years, $t(1334)=5.51, p<.001$ (Figure 1). Males were more likely to have an alcohol- or substance abuse-related CC compared to females $(\mathrm{OR}=16.9,95 \%$ CI: 9.1-31.3). The most prevalent CC among both female and male chronic high frequency users was "pain", occurring in $42 \%$ of visits $(n=21,993)$ and among $98 \%$ of MRNs $(n=1,309)$. One-third (34\%) of high frequency users had at least one visit with a $\mathrm{CC}$ relating to a mental health disorder while one-quarter (25\%) had at least one visit related to a dental complaint.

\section{Conclusions}

Chronic high frequency users of emergent health care services seek care for a variety of health complaints from chronic and acute illness to mental and oral health. Based on this initial assessment a portion of visits by highly frequent users could be directed to other health care services for appropriate treatment. Findings in this study support that of previous research which found that male high frequency ED users were older and had a higher rate of alcohol and substance abuse than female high frequency users ${ }^{3}$. Major limitations of this study were 1) health insurance coverage information was not accessible, which could assist to further characterize this health care-seeking population and 2) it was not possible to identify high frequency users that sought care across multiple facilities. Future research is needed to determine how chronic high frequency ED users differ from non-chronic users to establish potential focuses for interventions to reduce their burden on emergent health care services.

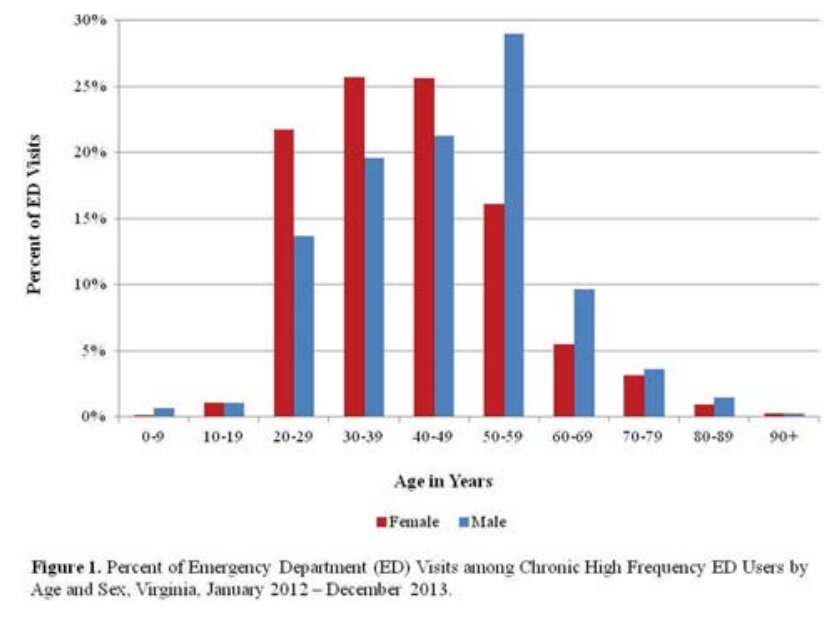

\section{Keywords}

ED utilization; frequent ED users; syndromic surveillance

\section{References}

1. Virginia Health Information, Inc. Annual Licensure Survey Data for Acute Care Hospital Emergency Departments, 2000-2012.

2. Castillo E, et al. Factors Associated With Frequent Users of Emergency Department Resources. Annals of Emer Med. 2012;60(4S):S32.

3. LaCalle E, et al. High-frequency users of emergency department care. J Emer Med. 2013;44(6):1167-1173.

*Erin E. Austin

E-mail: erin.austin@vdh.virginia.gov 